\title{
Synthesis and cytotoxic activity of new pyrazolo[1,5-a]pyrimidines and determination of pyrimidine regiospecific ring formation with 2D NMR
}

\author{
Eman K. A. Abdelall* and John N. Philoppes \\ Department of Pharmaceutical Organic Chemistry, Faculty of Pharmacy, Beni-Suef \\ University, Beni-Suef 62514, Egypt \\ E-mail:eman.ahmed@pharm.bsu.edu.eg
}

DOI: http://dx.doi.org/10.3998/ark.5550190.p009.743

\begin{abstract}
Novel pyrazolo[1,5-a]pyrimidines (9a, 9b, and 10a-c) were synthesized in high and efficient yields. Their pathway involves the formation of $N, S$ - ketene derivatives $(7 \mathbf{a}$ and $7 \mathbf{b})$ that reacted with hydrazine hydrate to get the key intermediate aminopyrazolo derivatives $(\mathbf{8 a}$ and $\mathbf{8 b})$. These aminopyrazoles were further reacted with either acetylacetoneor $\beta$-ketoesters resulting in the targeted pyrazolopyrimidines (9a, 9b, and 10a-c). All prepared compounds were fully characterized by spectral methods and the cyclization of 10a-d was proved by 2D NMR such as HMBC, HSQC and NOESY. The targeted pyrazolopyrimidines were subjected to in vitro anticancer screening and all of them showed promising cytotoxic activity when compared to doxorubicin. Compound 10d was the most active with $\mathrm{IC}_{50}=1.98,2.20$ and $2.61 \mu \mathrm{M}$ against MCF-7, BT474 and A549 cancer cell lines.
\end{abstract}

Keywords: Benzothiazole; 2D NMR; pyrazolopyrimidines; anticancer; MCF-7

\section{Introduction}

The development of new anticancer agents was a major area of research during the last decade. Instead of that complete over control of cancer has not been achieved yet. ${ }^{1}$ Beside to surgery, chemotherapy is still one of the most important medical options. Reasonably, many researches are directed to develop a new anti-cancer agent that targeting cancer cells and also with a low toxicity to normal cells. ${ }^{2-4}$ In addition, pyrazolo-pyrimidine base scaffolds are belonging to a biologically active class as they are structurally related to intrinsic purine bases. Obviously, several pyrazolopyrimidine models as compounds 1-3 (Fig.1) were reported to possess anticancer and cytotoxic activity. ${ }^{5-7}$ Compound 2 was reported to be an effective anti-tumour agent and a scaffold to adenosine (ATP) binding receptor in several kinases. ${ }^{6}$ Moreover, various 
synthetic methods of pyrazolopyrimidines have been described, ${ }^{7-11}$ and one of these methods involves the formation of ketene-S,S or $N, S$ - acetals. Such ketenes reacted with a bi-nucleophilic reagent such as hydrazine or substituted hydrazine to get the key intermediates 5-aminopyrazoles. $^{7}$

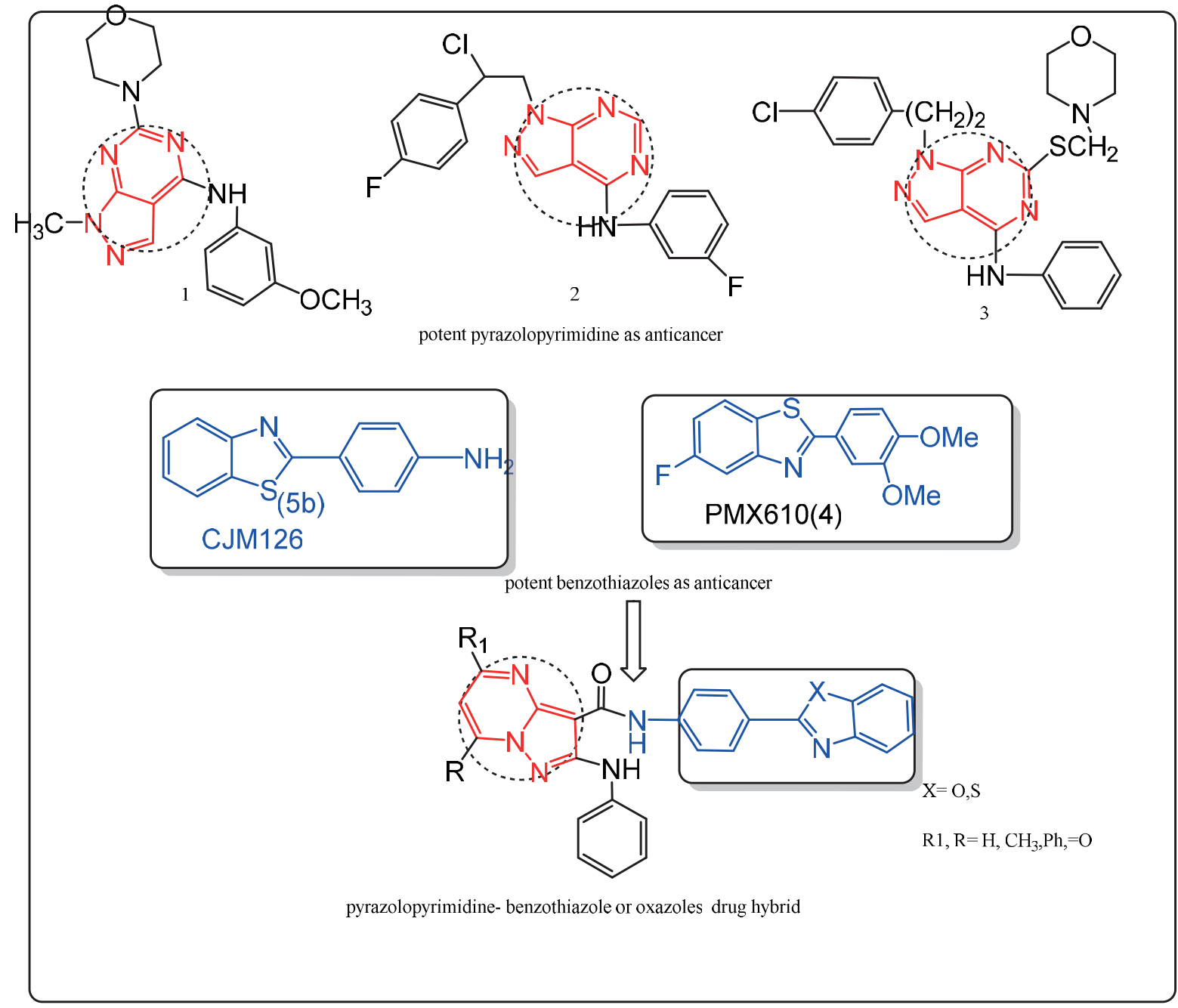

Figure1. Showing different potent anti-cancers: substituted pyrazolopyrimidines (1-3), and 2(3,4-dimethoxyphenyl)-5-fluorobenzothiazole (PMX610) (4) [4-(1,3-benzothiazol-2yl)phenyl]amine (5b) as anticancer agent and the design of new pyrazolopyrimidne benzothiazole or oxazoles hybrid drugs.

Such intermediates are versatile reagents for building more substituted pyrazolopyrimidines by further condensation with either diketones or $\beta$-ketoesters. In addition, several benzothiazole derivatives as [4-(1,3-benzothiazol-2-yl)phenyl]amine (CJM126)(5b) and 2- 
(3,4-dimethoxyphenyl)-5-fluorobenzothiazole (PMX610) ${ }^{12-15}$ are famous with their potency that enable them to be effective anti-cancer agents. Based on these findings, and as a continuation of previous work, ${ }^{16-20}$ we synthesized new expected drug hybrid of two active moieties pyrazolopyrimidine and benzothiazole or oxazole. The main objective of the research is to synthesize two series (9a,b) and (10a-d) in a convenient method as a new combination of pyrazolpyrimidine based scaffold substituted with benzothiazole or oxazole. This method starts by converting of either 4-aminophenyl-benzothiazole or oxazole to their corresponding 5aminopyrazole derivatives as a common precursor to pyrazolopyrimidines when reacted with diketones or $\beta$-ketoesters. These series of hybrids might be of more synergistic activity against MCF-7, BT474 and A549 cancer cell lines than the initial entities. Also, one of research objectives is directed toward exploring the mechanism of pyrazolopyrimidine cyclization through reaction of 5-aminopyrazole and $\beta$-ketoesters.

\section{Results and Discussion}

The reaction of amines 5a or $\mathbf{5} \mathbf{b}^{14}$ with ethyl cyanoacetate in DMF gave 2-cyano- $N$-substituted acetamido derivatives $6 \mathbf{a}$ and $\mathbf{6 b}$. The structure of $\mathbf{6 a}$ was confirmed by its ${ }^{1} \mathrm{H}$ NMR that showed $\left(\mathrm{CH}_{2} \mathrm{C} \equiv \mathrm{N}\right)$ at $\delta 3.97$. 6a and $\mathbf{6 b}$ were further reacted with phenylisothiocyanate and methyl iodide in the presence of $\mathrm{KOH}$ giving the $N, S$-ketene acetal derivatives $7 \mathbf{a}$ and $\mathbf{7 b}$.

Structure 7a was elucidated by its IR spectrum that showed two NH peaks at 3347 and 3291 $\mathrm{cm}^{-1}$ as well as a $\mathrm{C} \equiv \mathrm{N}$ band at $2179 \mathrm{~cm}^{-1}$ and also its ${ }^{1} \mathrm{H}$ NMR spectrum that exhibited a singlet at $\delta 2.28 \mathrm{ppm}\left(\mathrm{SCH}_{3}\right)$ and two $\mathrm{D}_{2} \mathrm{O}$ exchangeable peaks at $\delta 9.97$ and 11.55 corresponding to (NHCO) and (NH phenyl) respectively. Also, ${ }^{13} \mathrm{C}$ NMR of 7a confirm the appearance of signals for $\left(\mathrm{S}_{-} \mathrm{CH}_{3}\right), \beta$-ketene and $\alpha$-ketene at $\delta 16.89,74.35,167.91$ in sequence. These keteneacetals $7 \mathbf{a}$ and $\mathbf{7 b}$ were cyclized upon reaction with hydrazine in ethanol resulting in $\mathbf{8 a}$ and $\mathbf{8 b}$. The structure $\mathbf{8}$ is postulated through its spectral data and subsequent reaction. The ${ }^{1} \mathrm{H}$ NMR of $\mathbf{8 a}$ showed a singlet $\mathrm{D}_{2} \mathrm{O}$ exchangeable peak at $\delta 6.11$ corresponding to $\left(\mathrm{NH}_{2}\right)$ group (Scheme1).

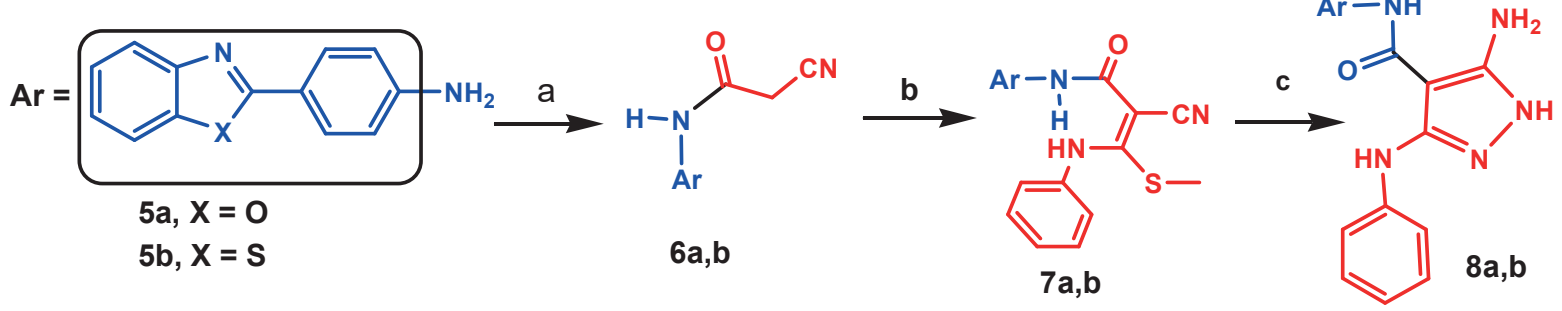

Scheme 1.Synthesis of 5-amino-pyrazoles 8a and 8b, Reagents and conditions: (a) DMF, $6 \mathrm{~h}$. , (b) $2 \mathrm{M} \mathrm{KOH}$, r.t, DMF, PhNCS, MeI, 6h., (c) $\mathrm{NH}_{2} \mathrm{NH}_{2}$, EtOH, 3 h. 
Then, these 5-aminopyrazoles were cyclized to the target compounds $9 \mathbf{a}$ and $\mathbf{9 b}$ through their reaction with 2,4-pentanedione in boiling acetic acid. With a symmetric diketone, only one compound was formed, the reaction proceeded that either $\mathrm{NH}$ or $\mathrm{NH}_{2}$ reacted with any carbonyl followed by condensation and removal of 2 moles of water giving $9 \mathbf{a}$ and $\mathbf{9 b}$ (Scheme 2).

The structure 9 was elucidated by elemental analysis and spectral data. Its IR revealed the disappearance of the cyano group and ${ }^{1} \mathrm{H}$ NMR spectrum of $9 \mathbf{b}$ showed singlets at $\delta 2.65,2.69$ for two $\left(\mathrm{CH}_{3}\right)$ while pyrimidine $\mathrm{H}-5$ appeared at $6.80-7.00$ as a multiplet with $\mathrm{H}-4$ of NHphenyl (Scheme 2).

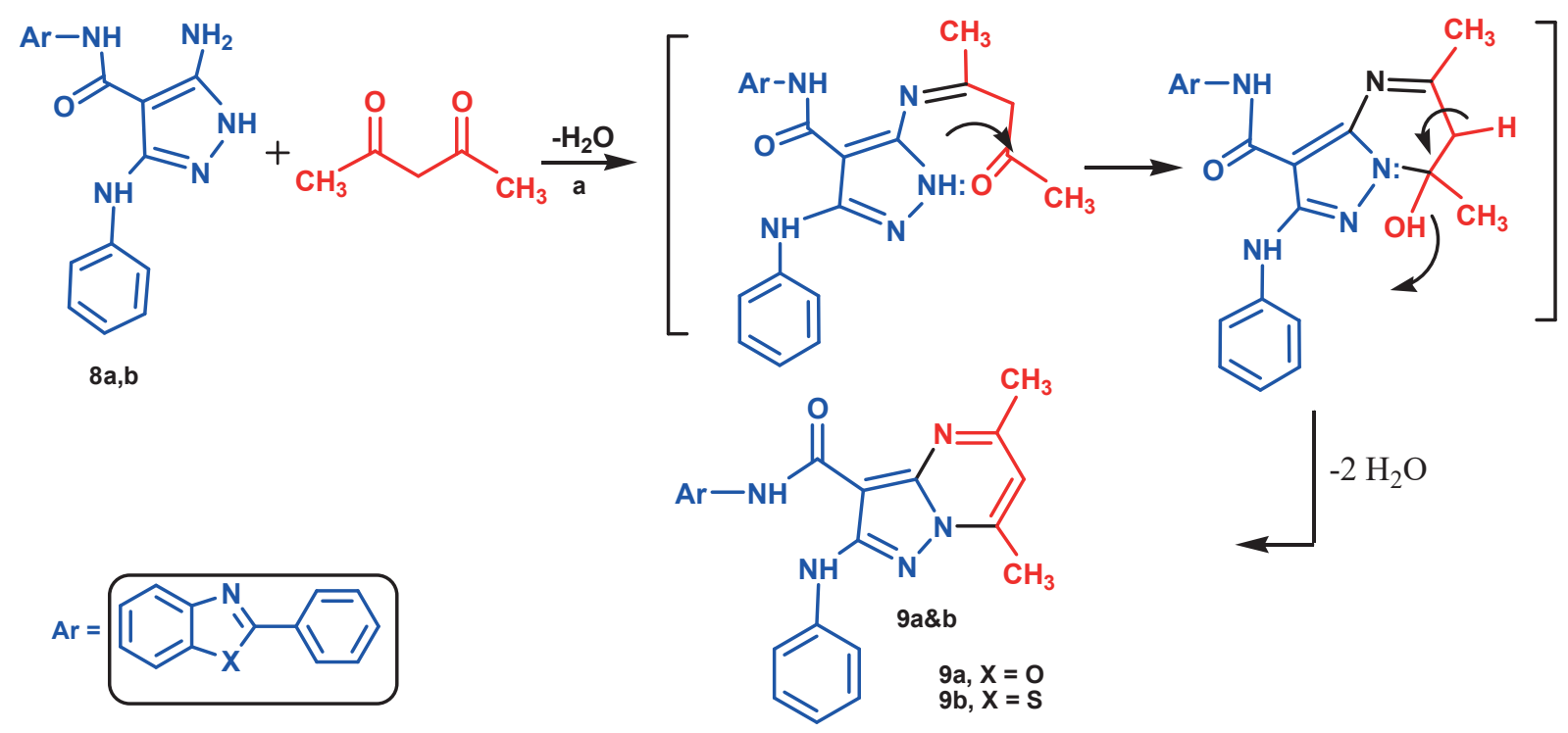

Scheme 2. preperation of of dimethylpyrazolo[1,5-a]pyrimidine 9a and 9b, Reagent and conditions: (a) AcOH, Reflux, $3 \mathrm{~h}$.

Additionally, the 5-aminopyrazoles 8a,b were reacted with $\beta$-ketoesters such as ethyl acetoacetate or ethyl 3-oxo-3-phenylpropanoate resulting in pyrazolopyrimidine compounds 10a-d (scheme3). The structure 10a-d was confirmed by elemental analysis, spectral data and MO calculation (Table1).

Theoretical MO calculations of heat of formation and bend energy (energy required to bend all bonds in a molecule that could be a measure of its stability) was done by using Chemdraw ultra and MM2 property revealed that $\mathbf{1 0 a}-\mathbf{d}$ is more stable in form $\mathbf{A}$ than $\mathbf{B}$. The MO caluculation of $(\mathbf{1 0 a}, \mathrm{A})$ showed a heat of formation $=-339.40086 \mathrm{Kcal} / \mathrm{mol}$ and bend energy $=$ $19.8561 \mathrm{Kcal} / \mathrm{mol}$, while in form $\mathbf{B}$ a heat of formation and bend energy $=-369.39 \mathrm{Kcal} / \mathrm{mol}$ and $20.508 \mathrm{Kcal} / \mathrm{mol}$ were found respectively. The previous result indicates that form $\mathbf{A}$ is more favorable than B. Practically, for 10a and due to lack of formation of a single crystal analysis, the structure of 10a was illustrated using HSQC, HMBC and NOESY techniques (Fig. 2). Upon studying 3D model of $\mathbf{1 0 a}$ in either form $\mathbf{A}$ or $\mathbf{B}$, form $\mathbf{A}$ showed a correlation between the 
protons of methyl group and both of pyrimidine $\mathrm{H}$, and $\mathrm{C}-4 \mathrm{H}$ of $\mathrm{NH}-$ phenyl in a distance within the range that appeared in the NOESY experiment (Fig. 2), while form $\mathbf{B}$ does not show these correlations. NOESY scan confirmed that 10a is in form $\mathbf{A}$ and not $\mathbf{B}$.

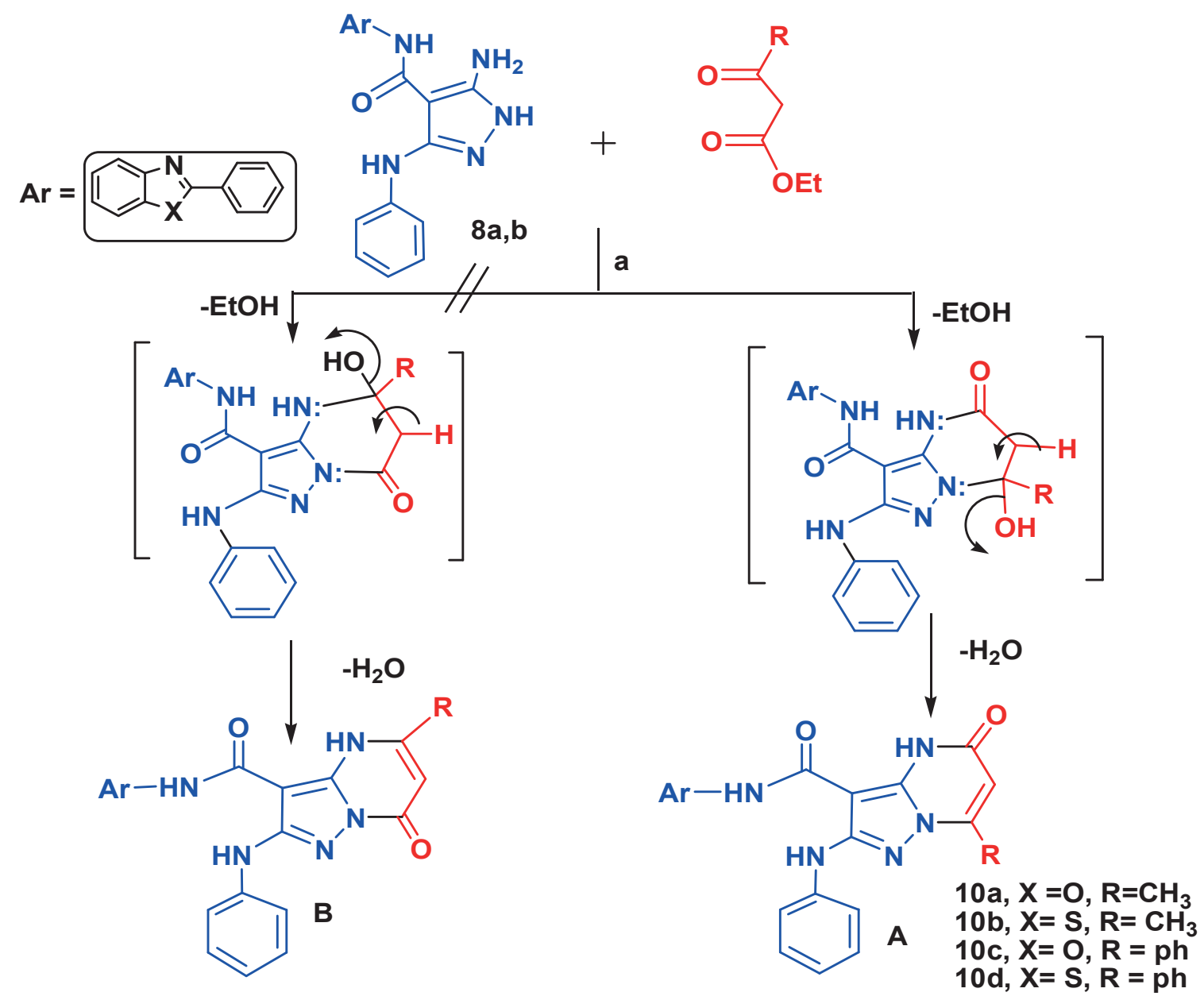

Scheme 3. Synthesis of substituted pyrazol[1,5-a]pyrimidines 10a-d. Reagent and condition: ethyl acetoacetate or ethyl 3-oxo-3-phenylpropanote, $\mathrm{AcOH}$, Reflux, $5 \mathrm{~h}$. 


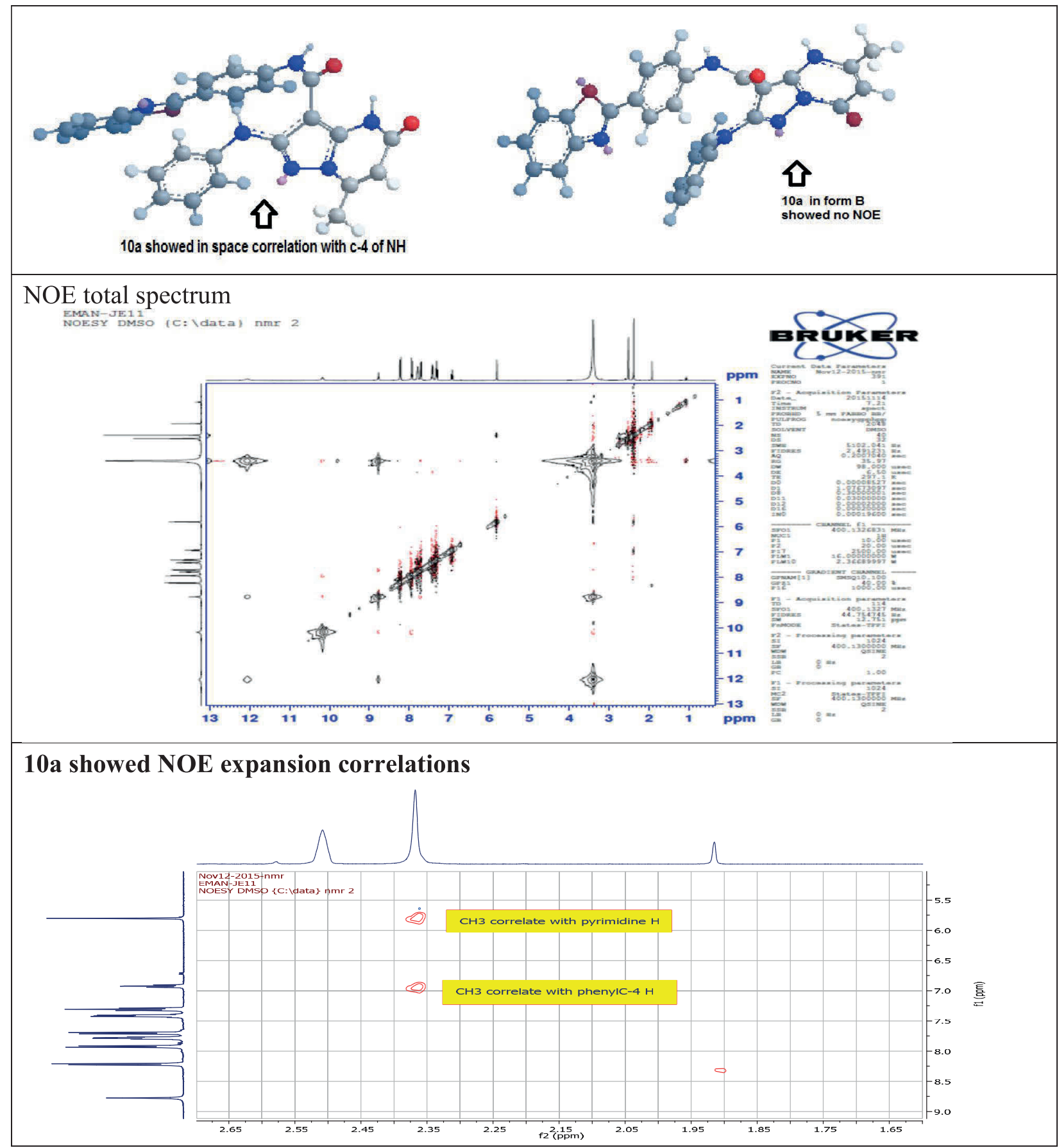

Figure 2. 3D representation of $10 a$ in form $\mathbf{A}$ and $\mathbf{B}$, NOESY scan of $10 a$. 
Table 1: Molecular orbital (M.O) calculation of heat of formation (HF) and bend energy (BE) for expected 10a-d (structure A or structure B)

\begin{tabular}{lll}
\hline & HF* $\backslash$ BE* of structure $\mathbf{A}$ & HF* $\backslash$ BE*of structure $\mathbf{B}$ \\
\hline $\mathbf{1 0 a}$ & HF: $-339.40086 \mathrm{Kcal} / \mathrm{mol}$ & HF: $-369.39 \mathrm{Kcal} / \mathrm{mol}$ \\
& BE: $19.8561 \mathrm{Kcal} / \mathrm{mol}$ & BE: $20.508 \mathrm{Kcal} / \mathrm{mol}$ \\
$\mathbf{1 0 b}$ & H F: $-359.7533 \mathrm{kcal} / \mathrm{mol}$ & $\mathrm{H} \mathrm{F}:-546.65 \mathrm{Kcal} / \mathrm{mol}$ \\
& BE: $22.9076 \mathrm{Kcal} / \mathrm{mol}$ & BE: $23.1722 \mathrm{Kcal} / \mathrm{mol}$ \\
$\mathbf{1 0 c}$ & HF: $-395.6209 \mathrm{kcal} / \mathrm{mol}$ & HF: $-426.6263 \mathrm{kcal} / \mathrm{mol}$ \\
& BE: $19.5831 \mathrm{Kcal} / \mathrm{mol}$ & BE: $20.107 \mathrm{Kcal} / \mathrm{mol}$ \\
$\mathbf{1 0 d}$ & H F: $-411.8006 \mathrm{kcal} / \mathrm{mol}$ & H F: $-679.98 \mathrm{Kcal} / \mathrm{mol}$ \\
& BE: $23.5574 \mathrm{Kcal} / \mathrm{mol}$ & BE: $23.5864 \mathrm{Kcal} / \mathrm{mol}$ \\
\hline
\end{tabular}

$\mathrm{HF}^{*}$ : heat of formation calculated after energy minimization of structures at $25^{\circ}$ using Chemdraw 3D ultra version8 and MM2 property BE*: Bend energy calculated at $25^{\circ}$

\section{Anticancer screening:}

Cytotoxic study was operated using MTT(3,4-dimethylthiazol-2-yl)-2,5-diphenyltetrazolium Bromide assay method. ${ }^{21}$ All tested compounds showed moderate to high cytotoxic activity against MCF-7, BT474 and A549 cell lines. The obtained data are listed Table 2. Most synthesized compounds (7a, 8a, 8b, 9a, 9b, 10a and 10c) showed moderate cytotoxic activity when compared with the reference. $7 \mathbf{b i s}$ the least potent one that exhibited a cytotoxic activity with $\mathrm{IC}_{50} 9.02,9.87,8.42 \mu \mathrm{M}$ against MCF-7, BT474 and A549 cell lines. Regarding compounds with an oxazole moiety only (6a and $\mathbf{8 a}$ ) they showed nearly the same moderate activity against all cell lines and they are more potent than $(\mathbf{6 b}$ and $\mathbf{8 b})$ with a benzothiazole moiety. The sight was directed to the compound of benzoythiazole or oxazole /pyrazolopyrimidine hybrids (9a, 9b and 10a-d). Their potency was ordered $\mathbf{1 0 d}>\mathbf{1 0 b}>\mathbf{9 b}>$ $\mathbf{9 a}>\mathbf{1 0 a}>\mathbf{1 0 c}$. The most potent is $\mathbf{1 0 d}$ with $\mathrm{IC}_{50} 1.98,2.2,2.61 \mu \mathrm{M}$ and its result is close to the reference doxorubicin with $\mathbf{I C}_{50} 1.72,1.81,1.21 \mu \mathrm{M}$ against MCF-7, BT474 and A549 cell lines. 10d is a pyrazolopyrimidine-benzothiazole hybrid and this reactivity could be related to, 1- high potency of benzothiazole moiety, 2- presence of $\mathrm{NH}$ tatuomeric $\mathrm{OH}$ that increase incidence of hydrogen bond (HB) with proposed receptor active site and 3- high lipophilicity due to phenyl moiety when compared to $\mathbf{1 0 b}$ which is also pyrazolopyrimidine-benzothiazole hybrid with a $\mathrm{CH}_{3}$ moiety. In addition $\mathbf{1 0 b}$ also showed higher potency activity with $\mathrm{IC}_{50}=2.01$, 2.36, $2.47 \mu \mathrm{M}$ against MCF-7, BT474 and A549 cell lines respectively. 
Table 2: $\mathrm{IC}_{50}$ of testing compounds and standard doxorubicin against MCF-7, BT474 and A549

\begin{tabular}{clll}
\hline \multirow{2}{*}{$\begin{array}{c}\text { Compound } \\
\text { no }\end{array}$} & \multicolumn{3}{c}{ IC $_{\mathbf{5 0}}(\boldsymbol{\mu M})$} \\
\cline { 2 - 4 } 6a & MCF-7 & BT474 & $\mathbf{A 5 4 9}$ \\
\hline 6b & $9.87 \pm 1.07$ & $6.74 \pm 1.03$ & $6.41 \pm 1.07$ \\
$\mathbf{8 a}$ & $5.21 \pm 0.77$ & $6.32 \pm 1.09$ & $6.27 \pm 1.02$ \\
$\mathbf{8 b}$ & $6.54 \pm 0.88$ & $6.98 \pm 1.09$ & $6.96 \pm 0.99$ \\
9a & $3.42 \pm 0.89$ & $4.26 \pm 0.77$ & $3.65 \pm 0.93$ \\
9b & $2.25 \pm 0.12$ & $2.11 \pm 0.87$ & $2.74 \pm 0.89$ \\
$\mathbf{1 0 a}$ & $2.73 \pm 0.70$ & $3.07 \pm 0.14$ & $2.91 \pm 0.78$ \\
$\mathbf{1 0 b}$ & $\mathbf{2 . 0 1} \pm \mathbf{0 . 4 0}$ & $\mathbf{2 . 3 6} \pm \mathbf{0 . 4 4}$ & $\mathbf{2 . 4 7} \pm \mathbf{0 . 3 8}$ \\
$\mathbf{1 0 c}$ & $5.47 \pm 0.80$ & $5.96 \pm 1.05$ & $6.36 \pm 0.94$ \\
$\mathbf{1 0 d}$ & $1.98 \pm 0.70$ & $2.20 \pm 0.50$ & $2.61 \pm 0.45$ \\
Doxorubicin & $1.72 \pm 0.7$ & $1.81 \pm 0.19$ & $1.21 \pm 0.33$ \\
\hline
\end{tabular}

Several benzothiazole and oxazole compounds $(\mathbf{6 a}, \mathbf{6 b}, \mathbf{7 a}, \mathbf{7 b}, \mathbf{8 a}$ and $\mathbf{8 b})$ were prepared and also pyrazolopyrimidine with a benzoxazole $(\mathbf{9 a}, \mathbf{1 0 a}$ and $\mathbf{1 0 c})$ those showed considerable cytotoxic activity while their corresponding pyrazolpyrimidines with a benzothiazole $(\mathbf{9 b}, \mathbf{1 0 b}$ and 10d) showed the highest activity. Such pyrazolopyrimidine-benzothiazole combination is the successful one that resulted in $10 \mathbf{b}$ and $10 \mathrm{~d}$ as the most active compounds. Structure elucidation of $10 \mathrm{a}$ with 2D and NOE excluded their structural form.

\section{Experimental Section}

General. Melting points were determined on an Electrothermal digital melting point apparatus and are uncorrected. IR spectra were recorded on an R 435 spectrophotometer (Middlton, Madison West, WI, USA) and values were reported in $\mathrm{cm}^{-1}$. ${ }^{1} \mathrm{H}-\mathrm{NMR}$ and ${ }^{13} \mathrm{C}-\mathrm{NMR}$ were carried out on Bruker Advance III $400 \mathrm{MHz}$ spectrophotometer (Bruker BioSpin AG, Fällanden, Switzerland) for ${ }^{1} \mathrm{H}$ and $100 \mathrm{MHz}$ for ${ }^{13} \mathrm{C}$ with BBFO Smart Probe and Bruker 400 AEON Nitrogen-Free Magnet, using TMS as an internal standard and chemical shifts were recorded in ppm on $\delta$ scale, Faculty of Pharmacy, Beni Suef University, Egypt. The electron impact (EI) mass spectra were recorded on a Hewlett Packard 5988 spectrometer (Palo Alto, CA, USA), Microanalyses for C, H and N were carried out on Perkin-Elmer 2400 analyzer (Perkin-Elmer, Norwalk, CT, USA) at the Micro analytical unit of Cairo University, Egypt, and all compounds were within $\pm 0.4 \%$ of the theoretical values. Thin-layer chromatography (TLC) was performed on Merck (Darmstadt, Germany ) TLC aluminium sheets silica gel 60 F254 with detection by UV 
quenching at $254 \mathrm{~nm}$ to follow the course of reactions and to check the purity of products. All reagents and solvents were purified and dried by standard techniques.

General procedure for synthesis of $\mathbf{6 a}, \mathbf{b}$. A solution of equivalent amount of ethyl cyanoacetate $(0.56 \mathrm{~g}, 5 \mathrm{mmol})$ with the corresponding amines $\mathbf{5 a}$ and $\mathbf{5 b}^{14}$ in dimethylformamide $(20 \mathrm{~mL})$ was heated under reflux for $6 \mathrm{~h}$. The reaction mixture was cooled and concentrated under vacuum to get the solid product which was crystallized from ethanol/dimethylformamide mixture (1:1).

N-(4-(Benzoxazol-2-ylphenyl)-2-cyanoacetamide (6a). Buff solid; Yield 87\%; mp 265-267 ${ }^{\circ} \mathrm{C}$; IR( $\left.\mathrm{KBr}, \mathrm{cm}^{-1}\right): 3439(2 \mathrm{NH}), 2199(\mathrm{C} \equiv \mathrm{N}), 1656(\mathrm{C}=\mathrm{O}) ;{ }^{1} \mathrm{H}$ NMR (DMSO-d $) \delta 3.97(\mathrm{~s}, 2 \mathrm{H}$, $\mathrm{CH}_{2}$ ), 7.39-7.42 (m, 2H, H-5 \& H-6 of benzoxazole), 7.75-7.80 (m, 4H, H-4 \&H-7 of benzoxazole and H-2\&H-6 of amidophenyl), 8.18 (d, J $8.4 \mathrm{~Hz}, 2 \mathrm{H}, \mathrm{H}-3 \& \mathrm{H}-5$ of amidophenyl), 10.64 (s, 1H, NHCO, $\mathrm{D}_{2} \mathrm{O}$ exchangeable); $\mathrm{MS}(\mathrm{m} / \mathrm{z}, \%): 278(\mathrm{M}+1,13.60 \%), 277\left(\mathrm{M}^{+}, 77.30 \%\right)$, $68\left(\mathrm{C}_{3} \mathrm{H}_{2} \mathrm{NO}^{+}, 100.00 \%\right)$; Anal. Calcd for $\mathrm{C}_{16} \mathrm{H}_{11} \mathrm{~N}_{3} \mathrm{O}_{2}$ (277.28): C, 69.31; H, 4.00; N, 15.15 . Found: C, 69.57; H, 3.85; N, 15.28.

N-(4-(Benzothiazol-2-ylphenyl)-2-cyanoacetamide (6b). Buff solid; Yield 82\%; mp 235-237 ${ }^{\circ} \mathrm{C}$; IR $\left(\mathrm{KBr}, \mathrm{cm}^{-1}\right): 3406(2 \mathrm{NH}), 2292(\mathrm{C} \equiv \mathrm{N}), 1640(\mathrm{C}=\mathrm{O}) ;{ }^{1} \mathrm{H}$ NMR (DMSO-d $\left.d_{6}\right) 3.96(\mathrm{~s}, 2 \mathrm{H}$, $\mathrm{CH}_{2}$ ), 7.42 (t $J 7.6 \mathrm{~Hz}, 1 \mathrm{H}, \mathrm{H}-6$-of benzothiazole), 7.53 (t $J 7.6 \mathrm{~Hz}, 1 \mathrm{H}, \mathrm{H}-5$ benzothiazole), 7.75 (d, $J 8.4$ Hz, 2H, H-2\&H-6 of amidophenyl), 8.01-8.13 (m, 4H, H-4\&H-7 of benzothiazole, $\mathrm{H}-3$ \& H-5 of amidophenyl), 10.60 (s, 1H, NHCO, $\mathrm{D}_{2} \mathrm{O}$ exchangeable); Anal. Calcd for $\mathrm{C}_{16} \mathrm{H}_{11} \mathrm{~N}_{3} \mathrm{OS}$ (293.34): C, 65.51; H, 3.78; N, 14.32. Found: C, 65.37; H, 3.95; N, 14.29.

General procedure for synthesis of $7 \mathbf{a}, 7 \mathbf{b}$. A solution of $\mathbf{6 a}$ or $\mathbf{6 b}(0.1 \mathrm{mmol})$, potassium hydroxide $(0.11 \mathrm{~g}, 2 \mathrm{mmol})$, phenyl isothiocyanate $(0.27 \mathrm{~g}, 2 \mathrm{mmol})$ in dimethylformamide (20 $\mathrm{mL})$ was stirred for $6 \mathrm{~h}$. To the reaction mixture methyl iodide was added $(0.28 \mathrm{~g}, 2 \mathrm{mmol})$ and the mixture stirred for $3 \mathrm{~h}$, the reaction mixture was concentrated under vacuum to get the product $7 \mathbf{a}$ or $\mathbf{7 b}$ that crystallized from ethanol/ dimethylformamide mixture (1:1)

$\mathrm{N}$-(4-Benzoxazol-2-ylphenyl)-2-cyano-3-methylsulfanyl-3-phenylaminoacetamide (7a). Yellow solid; Yield 85\%; mp 201-203 ${ }^{\circ} \mathrm{C}$; IR ( KBr, cm $\left.{ }^{-1}\right): 3447,3291(2 \mathrm{NH}), 2197(\mathrm{C} \equiv \mathrm{N}), 1629$ $(\mathrm{C}=\mathrm{O}) ;{ }^{1} \mathrm{H}$ NMR (DMSO- $\left.d_{6}\right) \delta 2.28\left(\mathrm{~s}, 3 \mathrm{H}, \mathrm{SCH}_{3}\right), 7.21-7.37$ (m, 1H, H-4 of NH-phenyl), 7.397.55(m, 6H, H-2, H-6, H-3, H-5-of NH-phenyl and H-5 \& H-6 of benzoxazole ), 7.74-7.78 ( m, $4 \mathrm{H}, \mathrm{H}-2 \& \mathrm{H}-6$ of amidophenyl and H-4\&H-7 of benzoxazole), 8.01(d $J 7.2,2 \mathrm{H}, \mathrm{H}-3 \& \mathrm{H}-5$ of amidophenyl), 9.97 (s,1H, $\mathrm{NH}\left(\mathrm{D}_{2} \mathrm{O}\right.$ exchangeable), $11.55\left(\mathrm{~s}, 1 \mathrm{H}, \mathrm{NH}\left(\mathrm{D}_{2} \mathrm{O}\right.\right.$ exchangeable) ${ }^{13} \mathrm{C}$ NMR (DMSO- $\left.d_{6}\right) \delta 16.89,74.35,111.23,118.90,120.02,121.11,121.56,123.96,125.26$, 125.67, 126.47, 128.31, 129.72, 139.14, 142.10, 142.33, 150.59, 162.66, 164.5, 167.91; Anal. Calcd for $\mathrm{C}_{24} \mathrm{H}_{18} \mathrm{~N}_{4} \mathrm{O}_{2} \mathrm{~S}$ (426.49): C, 67.59; H, 4.25; N, 13.14. Found: C, 67.37; H, 4.55; N, 13.38 .

$\mathrm{N}$-(4-Benzothiazol-2-ylphenyl)-2-cyano-3-methylsulfanyl-3-phenylaminoacetamide (7b). Red solid; Yield 82\%; mp 203-205 ${ }^{\circ} \mathrm{C}$; IR( $\left.\mathrm{KBr}, \mathrm{cm}^{-1}\right): 3440,3290(2 \mathrm{NH}), 2195(\mathrm{C} \equiv \mathrm{N}), 1625$ $(\mathrm{C}=\mathrm{O}) ;{ }^{1} \mathrm{H}$ NMR (DMSO-d $): \delta 2.44\left(\mathrm{~s}, 3 \mathrm{H}, \mathrm{SCH}_{3}\right), 7.21-7.23(\mathrm{~m}, 1 \mathrm{H}, \mathrm{H}-4$ of NH-phenyl), 7.31- 
7.48 (m, 6H, H-2, H-6, H-3, H-5 of NH-phenyl and H-5 \& H-6 of benzothiazole), 7.68 (d $J$ 8.4, 2H, H-2\&H-6 of amidophenyl), 7.96 (d $J 8.4,2 \mathrm{H}, \mathrm{H}-3$ \& H-5 of amidophenyl), 8.02 (m, 2H, H$4 \& \mathrm{H}-7$ of benzothiazole), $9.88\left(\mathrm{~s}, 1 \mathrm{H}, \mathrm{NH}, \mathrm{NHCO}\left(\mathrm{D}_{2} \mathrm{O}\right.\right.$ exchangeable), 11.57 (s, 1H, NH, NHphenyl ( $\mathrm{D}_{2} \mathrm{O}$ exchangeable), ${ }^{13} \mathrm{C}$ NMR (DMSO- $\left.d_{6}\right) \delta 16.90,71.01,111.25,121.29,122.75$, $123.06,124.00,125.76,126.50,127.07,128.12,128.31,129.72,134.76,141.66,146.80$, 154.09, 167.42, 168.25, 172.66; $\mathrm{MS}(\mathrm{m} / \mathrm{z}, \%): 443(\mathrm{M}+1,15.41 \%), 442\left(\mathrm{M}^{+}, 42.51 \%\right), 226$ $\left(\mathrm{C}_{13} \mathrm{H}_{10} \mathrm{~N}_{2} \mathrm{~S}^{+}, 100.00 \%\right.$ ); Anal.Calcd for $\mathrm{C}_{24} \mathrm{H}_{18} \mathrm{~N}_{4} \mathrm{OS}_{2}$ (442.56): C, 65.13; H, 4.10; N, 12.66 . Found: C, 64.98; H, 4.27; N, 12.45 .

General procedure for synthesis of $8 \mathbf{a}, \mathbf{8 b}$. A suspension of $7 \mathbf{a}$ or $7 \mathbf{b}(5 \mathrm{mmol})$ in ethanol (20 $\mathrm{ml})$ and hydrazine hydrate $(0.16 \mathrm{gm}, 5 \mathrm{mmol})$ was heated under reflux for $3 \mathrm{~h}$, the solid formed on hot was separated and crystallized from ethanol/dimethylformamide mixture (1:1).

5-Amino-3-phenylamino-1H-pyrazol-4-carboxylic acid (4-benzoxazol-2-ylphenyl)-amide (8a).Buff solid; Yield 73\%; mp 259-261 ${ }^{\circ} \mathrm{C}$; IR $\left(\mathrm{KBr}, \mathrm{cm}^{-1}\right)$ : 3438-3295 (3NH and $\left.\mathrm{NH}_{2}\right), 1661$ $(\mathrm{C}=\mathrm{O}) ; 1604(\mathrm{C}=\mathrm{N}) ;{ }^{1} \mathrm{H}$ NMR (DMSO-d $): \delta 6.11\left(\mathrm{~s}, 2 \mathrm{H}, \mathrm{NH}_{2}\right.$ 5-amino-pyrazole $\left(\mathrm{D}_{2} \mathrm{O}\right.$ exchangeable)), 6.76-7.92(m, 1H, H-4 of NH-phenyl), 7.18-7.43 (m, 6H, H-2, H-6, H-3, H5-of NH-phenyl and H-5 \& H-6 of benzoxazole), 7.74-7.77 (m, 4H,H-4 \&H-7 of benzoxazole and H-2\&H-6 of amidophenyl), 8.13 (d $J$ 8.4, 2H, H-3\&H-5of amidophenyl), 8.53 (s, 1H, NHphenyl ( $\mathrm{D}_{2} \mathrm{O}$ exchangeable $\left.)\right), 9.09\left(\mathrm{~s}, \mathrm{NH}, \mathrm{NHCO}\left(\mathrm{D}_{2} \mathrm{O}\right.\right.$ exchangeable $\left.)\right), 11.36(\mathrm{~s}, 1 \mathrm{H}, \mathrm{NH}, \mathrm{NH}-$ pyrazole $\left(\mathrm{D}_{2} \mathrm{O}\right.$ exchangeable)). MS (m/z,\%): $411(\mathrm{M}+1,6.34 \%), 410\left(\mathrm{M}^{+}, 22.54 \%\right), 210$ $\left(\mathrm{C}_{13} \mathrm{H}_{10} \mathrm{~N}_{2} \mathrm{O}^{+}, 100.00 \%\right)$; Anal.Calcd for $\mathrm{C}_{23} \mathrm{H}_{18} \mathrm{~N}_{6} \mathrm{O}_{2}$ (410.43):C, 67.31; H, 4.42; N, 20.48 . Found: C, 67.38; H, 4.65; N, 20.39.

5-Amino-3-phenylamino-1H-pyrazol-4-carboxylic acid (4-benzothiazol-2-ylphenyl)amide (8b). Buff solid; Yield 68\%; mp 254-256 ${ }^{\circ} \mathrm{C}$; IR( $\left.\mathrm{KBr}, \mathrm{cm}^{-1}\right): 3458-3261$ (3NH and $\mathrm{NH}_{2}$ ), 1644 $(\mathrm{C}=\mathrm{O}) ; 1592(\mathrm{C}=\mathrm{N}) ;{ }^{1} \mathrm{H}$ NMR (DMSO-d $): \delta 6.14\left(\mathrm{~s}, 2 \mathrm{H}, \mathrm{NH}_{2}, 5\right.$-aminopyrazole $\left(\mathrm{D}_{2} \mathrm{O}\right.$ exchangeable)), 6.80 (t $J 7.2 \mathrm{~Hz}, 1 \mathrm{H}, \mathrm{H}-4$ of NH-phenyl), 7.22 (t $J 7.2 \mathrm{~Hz}, 2 \mathrm{H}, \mathrm{H}-2 \& \mathrm{H}-6$ of NHphenyl), 7.32 (d $J 7.2,2 \mathrm{H}, \mathrm{H}-3$ \& H-5 of NH-phenyl), 7.51 (t $J 7.2 \mathrm{~Hz}, 1 \mathrm{H}, \mathrm{H}-6$ benzothiazole ),7.55 (t $J 7.2 \mathrm{~Hz}, 1 \mathrm{H}, \mathrm{H}-5$ benzothiazole), 7.74 (d J8.4, 2H, H-2\&H-6 of amidophenyl), 8.018.08 (m, 3H, H-3 \& H-5 of amidophenyl and H-7 of benzothiazole), 8.11(d, J 7.2, 1H, H-4 of benzothiazole), 8.57 (s, 1H, NH-phenyl ( $\mathrm{D}_{2} \mathrm{O}$ exchangeable), $9.07(\mathrm{~s}, 1 \mathrm{H}, \mathrm{NHCO}), 11.39(\mathrm{~s}, 1 \mathrm{H}$, NH-pyrazole ( $\mathrm{D}_{2} \mathrm{O}$ exchangeable)). Anal. Calcd for $\mathrm{C}_{23} \mathrm{H}_{18} \mathrm{~N}_{6} \mathrm{OS}$ (426.49): C, 64.77; H, 4.25; N, 19.70. Found: C, 64.98; H, 4.46; N, 19.89.

General procedure for synthesis of $(9 a$ and $9 b)$. A mixture of the appropriate aminopyrazole 8aor $\mathbf{8 b}(2 \mathrm{mmol})$ and acetylacetone $(0.2 \mathrm{~g}, 2 \mathrm{mmol})$ in glacial acetic acid $(15 \mathrm{~mL})$ was boiled under reflux for $3 \mathrm{~h}$. The resulting solid was collected and recrystallized from ethanol/DMF mixture (3:1) to achieve $\mathbf{9 a}$ or $\mathbf{9 b}$.

$N$-(4-(Benzo[d] oxazol-2-yl)phenyl)-5,7-dimethyl-2-(phenylamino)pyrazolo[1,5a]pyrimidine-3-carboxamide (9a).Yellowish white; Yield 62\%; mp 283-285 ${ }^{\circ} \mathrm{C}$; $\mathrm{IR}\left(\mathrm{KBr}, \mathrm{cm}^{-}\right.$ $\left.{ }^{1}\right): 3320,3222(2 \mathrm{NH}), 2919\left(\mathrm{CH}\right.$ aliphatic), $1669(\mathrm{C}=\mathrm{O}) ; 1595(\mathrm{C}=\mathrm{N}) ;{ }^{1} \mathrm{HNMR}\left(\mathrm{DMSO}-d_{6}\right): \delta$ 
$2.70\left(\mathrm{~s}, 3 \mathrm{H}, \mathrm{CH}_{3}\right), 2.77$ (s, 3H, $\left.\mathrm{CH}_{3}\right), 7.12(\mathrm{~s}, 1 \mathrm{H}, \mathrm{CH}$ pyrimidine), 7.12-7.16 (m,1H, H-4 of NHphenyl), 7.36-7.57 (m, 2H, H-4, H-2\&H-6 of NH-phenyl), 7.81 (d $J$ 7.6, 2H, H-3\&H-5 of NHphenyl), 8.03-8.14 (m, 8H, H-5 \& H-6 , H-4 \&H-7 of benzoxazole and H-2\&H-6, H-3\&H-5 of amidophenyl), 9.37 (s,1H, NHCO ( $\mathrm{D}_{2} \mathrm{O}$ exchangeable)), 10.49 (s, NH, NH-phenyl $\left(\mathrm{D}_{2} \mathrm{O}\right.$ exchangeable)), Anal. Calcd for $\mathrm{C}_{28} \mathrm{H}_{22} \mathrm{~N}_{6} \mathrm{O}_{2}$ (474.51): C, 70.87; H, 4.67; N, 17.71. Found: C, 71.11; H, 4.82; N, 17.76.

\section{$\mathrm{N}$-(4-(Benzo[d]thiazol-2-yl)phenyl)-5,7-dimethyl-2-(phenylamino)pyrazolo[1,5-}

a]pyrimidine-3-carboxamide (9b). Yellow solid; Yield 63\%; mp 251-253 ${ }^{\circ} \mathrm{C}$; $\mathrm{IR}\left(\mathrm{KBr}, \mathrm{cm}^{-1}\right)$ : 3312, 3229 (2NH), 2921 (CH aliphatic), $1666(\mathrm{C}=\mathrm{O}) ; 1599(\mathrm{C}=\mathrm{N}) ;{ }^{1} \mathrm{H}$ NMR (DMSO- $\left.d_{6}\right): \delta 2.65$ (s, 3H, $\left.\mathrm{CH}_{3}\right), 2.69\left(\mathrm{~s}, 3 \mathrm{H}, \mathrm{CH}_{3}\right), 6.80-7.00(\mathrm{~m}, 2 \mathrm{H}, \mathrm{H}-4$ of NH-phenyl and $\mathrm{CH}$ pyrimidine), 7.37 ( $J 8.0 \mathrm{~Hz}, 2 \mathrm{H}, \mathrm{H}-2 \& \mathrm{H}-6$ of NH-phenyl), 7.43 (t $J 7.2 \mathrm{~Hz}, 1 \mathrm{H}, \mathrm{H}-6$ benzothiazole ), 7.51-7.53 (t $J 8.0 \mathrm{~Hz}, 1 \mathrm{H}, \mathrm{H}-5$ benzothiazole), 7.76 (d $J .2 .0 \mathrm{~Hz}, 2 \mathrm{H}, \mathrm{H}-3$ \&H-5 of NHphenyl), 7.99 (d $J$ $8.8 \mathrm{~Hz}, 2 \mathrm{H}, \mathrm{H}-2 \& \mathrm{H}-6$ of amidophenyl), 8.01 (d $J 8.0 \mathrm{~Hz}, 1 \mathrm{H}, \mathrm{H}-7$ of benzothiazole), 8.06-8.10 (m, 3H, H-3\&H-5 of amidophenyl and $\mathrm{H}-4$ of benzothiazole), $9.31\left(\mathrm{~s}, 1 \mathrm{H}, \mathrm{NHCO}\left(\mathrm{D}_{2} \mathrm{O}\right.\right.$ exchangeable)), 10.28 (s, NH, NH-phenyl ( $\mathrm{D}_{2} \mathrm{O}$ exchangeable)), MS $(\mathrm{m} / z, \%) 491(\mathrm{M}+1$, $10.39 \%), 490\left(\mathrm{M}^{+}, 29.97 \%\right), 265\left(\mathrm{C}_{14} \mathrm{H}_{11} \mathrm{~N}_{5} \mathrm{O}^{+}, 100.00 \%\right)$; Anal. Calcd for $\mathrm{C}_{28} \mathrm{H}_{22} \mathrm{~N}_{6} \mathrm{OS}$ (490.58): C, 68.55; H, 4.52; N, 17.13. Found: C, 68.33; H, 4.38; N, 17.16.

General procedure for synthesis of $\mathbf{1 0 a - d . ~ A ~ m i x t u r e ~ o f ~ t h e ~ a p p r o p r i a t e ~ a m i n o p y r a z o l e ~ 8 a ~ o r ~}$ $\mathbf{8 b}(2 \mathrm{mmol})$ and ethylacetoacetate or ethyl 3-oxo-3-phenylpropanoate $(2 \mathrm{mmol})$ in glacial acetic acid $(15 \mathrm{~mL})$ was boiled under reflux for $5 \mathrm{~h}$. The resulting solid was collected and recrystallized from ethanol/DMF mixture to afford 10a-d.

$N$-(4-(Benzo[d]oxazol-2-yl)phenyl)-4,7-dihydro-5-methyl-7-oxo-2-(phenylamino)pyrazolo [1,5-a]pyrimidine-3-carboxamide (10a). Brown solid; Yield 65\%; mp 298-300 ${ }^{\circ} \mathrm{C}$; $\mathrm{IR}(\mathrm{KBr}$, $\mathrm{cm}^{-1}$ ): 3471, 3287, $3182(3 \mathrm{NH}), 2935$ (CH aliphatic), 1657, $1622(2 \mathrm{C}=\mathrm{O}),{ }_{1}^{1} \mathrm{H}$ NMR (DMSO- $\left.d_{6}\right)$ : $\delta 2.37$ (s, 3H, $\left.\mathrm{CH}_{3}\right), 5.80$ (s, 1H, CH Pyrimidine), 7.29 (t J 8.2 Hz, 1H, H-4), 7.40-7.42 ( m, $J 4.0 \mathrm{~Hz}, 2 \mathrm{H}, \mathrm{H}-5 \& \mathrm{H}-6$ of benzoxazole and H-2\& H-6 of NH-phenyl)), $7.71(\mathrm{~d} J 8.0 \mathrm{~Hz}, 2 \mathrm{H}, \mathrm{H}-$ $3 \& \mathrm{H}-5$ of NH-phenyl), 7.78 (t $J 4.0 \mathrm{~Hz}, 2 \mathrm{H}, \mathrm{H}-4 \& \mathrm{H}-7$ of benzoxazole), 7.92 (d $J 8.2 \mathrm{~Hz}, 2 \mathrm{H}$, H-2\&H-6 of amidophenyl), 8.22 (d $J 8.2 \mathrm{~Hz}, 2 \mathrm{H}, \mathrm{H}-3 \& \mathrm{H}-5$ of amidophenyl), 8.77 (s, 1H, NH, NH pyrazole $\left(\mathrm{D}_{2} \mathrm{O}\right.$ exchangeable $\left.)\right),\left(\mathrm{s}, 1 \mathrm{H}, \mathrm{NHCO}\left(\mathrm{D}_{2} \mathrm{O}\right.\right.$ exchangeable $\left.)\right), 12.05(\mathrm{~s}, 1 \mathrm{H}, \mathrm{NH}$ of NH-phenyl ( $\mathrm{D}_{2} \mathrm{O}$ exchangeable), ${ }^{13} \mathrm{C}$ NMR (DMSO- $\left.d_{6}\right) \delta 21.53,89.00,98.84,110.73,111.25$, $117.68,120.01,121.03,121.38,125.30,125.70,128.43,129.28,135.40,141.38,142.12$, $142.84,150.59,153.24,155.31,162.10,162.74,172.50, \mathrm{MS}(\mathrm{m} / \mathrm{z}, \%) 477(\mathrm{M}+1,9.94 \%), 476$ $\left(\mathrm{M}^{+}, 29.04 \%\right), 210\left(\mathrm{C}_{13} \mathrm{H}_{10} \mathrm{~N}_{2} \mathrm{O}^{+}, 100.00 \%\right)$; Anal.Calcd for $\mathrm{C}_{27} \mathrm{H}_{20} \mathrm{~N}_{6} \mathrm{O}_{3}$ (476.49): C, 68.06; $\mathrm{H}$, 4.23; N, 17.74. Found: C, 67.88; H, 3.95; N, 17.88.; HMBC, HSQC and NOESY scan are in supplementary materials.

$N$-(4-(Benzo[d] thiazol-2-yl)phenyl)-4,7-dihydro-5-methyl-7-oxo-2-(phenylamino)pyrazolo [1,5-a]pyrimidine-3-carboxamide (10b). Buff solid; Yield 69\%; mp 284-286 ${ }^{\circ} \mathrm{C}$; IR IR(KBr,cm $\left.{ }^{-1}\right): 3438,3280,3120(3 \mathrm{NH}), 1674,1642(2 \mathrm{C}=\mathrm{O}) ;{ }^{1} \mathrm{H}$ NMR (DMSO $): \delta 2.37(\mathrm{~s}, 3 \mathrm{H}$, $\mathrm{CH}_{3}$ ), 5.81 (s, 1H, pyrimidine H), 6.92 (t $J 7.60 \mathrm{~Hz}, 1 \mathrm{H}, \mathrm{H}-4$ of NH-phenyl), 7.31 (t $J 7.60 \mathrm{~Hz}$, 
2H, H-3\& H-5 of NH-phenyl), 7.45 (t $J 7.2 \mathrm{~Hz}, 1 \mathrm{H}, \mathrm{H}-6$ benzothiazole), 7.55 (t $J 7.2 \mathrm{~Hz}, 1 \mathrm{H}$, H-5 benzothiazole), 7.70 (d $J 7.6 \mathrm{~Hz}, 2 \mathrm{H}, \mathrm{H}-2 \& \mathrm{H}-6$ of NH-phenyl), 7.89(d $J 8.40 \mathrm{~Hz}, 2 \mathrm{H}, \mathrm{H}-$ 2\&H-6 of amidophenyl), 8.04 (d $J 7.2 \mathrm{~Hz}, 1 \mathrm{H}, \mathrm{H}-7$ benzothiazole), 8.11-8.15 (m, 3H, H-3\&H5 of amidophenyl and $\mathrm{H}-4$ of benzothiazole), 8.77 ( $\mathrm{s}, 1 \mathrm{H}, \mathrm{NH}$ pyrazole $\left(\mathrm{D}_{2} \mathrm{O}\right.$ exchangeable $)$ ), 10.10 (s,1H, NHCO ( $\mathrm{D}_{2} \mathrm{O}$ exchangeable)), 12.04 (s, 1H, NH, NH-phenyl ( $\mathrm{D}_{2} \mathrm{O}$ exchangeable)); MS (m/z,\%) $539(\mathrm{M}+1,0.10 \%), 538\left(\mathrm{M}^{+}, 0.12 \%\right), 80\left(\mathrm{C}_{3} \mathrm{H}_{2} \mathrm{~N}_{3}{ }^{+}, 100.00 \%\right)$; Anal.Calcd for $\mathrm{C}_{32} \mathrm{H}_{22} \mathrm{~N}_{6} \mathrm{O}_{3}$ (538.56): C, 71.37; H, 4.12; N, 15.60. Found: C, 71.53; H, 3.90; N, 15.78.

$\mathrm{N}$-(4-(Benzo[d]oxazol-2-yl)phenyl)-4,7-dihydro-5-phenyl-7-oxo-2-(phenylamino)pyrazolo [1,5-a] pyrimidine-3-carboxamide (10c). Buff solid; Yield 62\%; mp 287-289 ${ }^{\circ} \mathrm{C}$; IR $\left(\mathrm{KBr}, \mathrm{cm}^{-}\right.$ $\left.{ }^{1}\right): 3439,3288,3185(3 \mathrm{NH}), 2925$ (CH aliphatic), 1657,1624 (2C=O); ${ }^{1} \mathrm{H}$ NMR (DMSO- $\left.d_{6}\right): \delta$ 6.30 (s,1H, CH pyrimidine), 6.95 (m, 1H, H-4 of NH-phenyl), 7.33-7.42 (m, 4H, H-3\& H-5 of NH-phenyl and H-5\&H-6 of benzoxazole), 7.60-7.61(m, 3H, H-3, H-4 and H-5 pyrimidine phenyl), 7.78-7.79 (d $J 6.0 \mathrm{~Hz}, 4 \mathrm{H}, \mathrm{H}-2 \& \mathrm{H}-6$ of NH-phenyl and H-2\&H-6 of pyrimidine phenyl), 7.97-8.00 (m,4H, H-4 \& H-7 of benzoxazole and H-2\&H-6 of amidophenyl), 8.23 (d $J$ $8.2,2 \mathrm{H}, \mathrm{H}-3 \& \mathrm{H}-5$ of amidophenyl), 8.99 (s, 1H, NH, $\mathrm{NHCO}\left(\mathrm{D}_{2} \mathrm{O}\right.$ exchangeable $\left.)\right), 10.55(\mathrm{~s}, 1 \mathrm{H}$, $\mathrm{NH}$ phenyl $\left(\mathrm{D}_{2} \mathrm{O}\right.$ exchangeable $\left.)\right) ;{ }^{13} \mathrm{C}$ NMR $\left(\mathrm{DMSO}-d_{6}\right) \delta 19.40,88.75,98.84,117.40,117.70$, $121.07,121.22$, 122.74, 123.04, 125.78, 127.11, 128.21, 129.30, 134.75, 136.66, 141.40, 142.31, 153.24, 154.10, 155.31, 162.05, 164.70, 167.50; Anal.Calcd for $\mathrm{C}_{27} \mathrm{H}_{20} \mathrm{~N}_{6} \mathrm{O}_{2} \mathrm{~S}$ (492.55): C, 65.84; H, 4.09; N, 17.06. Found: C, 66.05; H, 3.85; N, 16.96 .

$\mathrm{N}$-(4-(benzo $[d]$ thiazol-2-yl)phenyl)-4,7-dihydro-5-phenyl-7-oxo-2-(phenylamino)pyrazolo [1,5-a]pyrimidine-3-carboxamide (10d). Buff solid; Yield 64\%; mp 287-289 ${ }^{\circ} \mathrm{C} ; \mathrm{IR}\left(\mathrm{KBr}, \mathrm{cm}^{-}\right.$ $\left.{ }^{1}\right): 3414,3281,3180(3 \mathrm{NH}), 1666,1632(2 \mathrm{C}=\mathrm{O}) ;{ }^{1} \mathrm{H}$ NMR (DMSO- $\left.d_{6}\right): \delta 6.31(\mathrm{~s}, 1 \mathrm{H}$, pyrimidine H), 6.92 (t $J 8.00 \mathrm{~Hz}, 1 \mathrm{H}, \mathrm{H}-4$ of NH-phenyl), 7.34 (t $J 7.60 \mathrm{~Hz}, 2 \mathrm{H}, \mathrm{H}-2$ \& H-6 of NH-phenyl), 7.45 (t $J 7.2 \mathrm{~Hz}, 1 \mathrm{H}, \mathrm{H}-6$ benzothiazole), 7.53-7.67 (m, 4H, H-5 benzothiazole and H-3\&H-5 of pyrimidine phenyl), 7.62 (d J 7.6 Hz, 2H, H-3\& H-5 of NH-phenyl), 7.92 (d J 8.40 Hz, 2H, H$2 \& \mathrm{H}-6$ of pyrimidine phenyl), 8.03-8.05(m, $4 \mathrm{H}, \mathrm{H}-7 \& \mathrm{H}-4$ benzothiazole and H-2\&H-6 of amidophenyl), 8.15 (d $J 8.2 \mathrm{~Hz}, 2 \mathrm{H}, \mathrm{H}-3 \& \mathrm{H}-5$ of amidophenyl) 8.98 (s, $1 \mathrm{H}, \mathrm{NHCO}\left(\mathrm{D}_{2} \mathrm{O}\right.$ exchangeable)), 10.60 (s,1H,NH, NH-phenyl ( $\mathrm{D}_{2} \mathrm{O}$ exchangeable)), pyrazole $\mathrm{NH}$ not appeared . Anal.Calcd for $\mathrm{C}_{32} \mathrm{H}_{22} \mathrm{~N}_{6} \mathrm{O}_{2} \mathrm{~S}$ (554.62): C, 69.30; H, 4.00; N, 15.15. Found: C, 69.43; H, 3.87; N, 14.94.

Anti-tumour activity. Three human cancer cell lines [Human Breast Adenocarcinoma (MCF7) and BT474 and Non-Small Cell Lung Cancer (A549)] were obtained from the American Type Culture Collection. Cells were maintained in Dulbecco's Modified Eagle's Medium (DMEM:

Gibco, USA) supplemented with $\mathbf{1 0 \%}$ fetal bovine serum (Gibco), penicillin/streptomycin (Gibco). Cells were incubated at $37{ }^{\circ} \mathrm{C}$ in a humidified incubator containing $5 \% \mathrm{CO}_{2}$. Cell viability count was assessed using MTT (3-(4,5-dimethylthiazol-2-yl)-2,5-Diphenyltetrazolium Bromide) (Sigma-Aldrich, St. Louis, MO, USA) in 6 


\section{Acknowledgements}

We are grateful to Pharmaceutical Organic Chemistry for kind support during progressing of this research.

\section{References}

1. Lesyk, R.; Validzimirska, O.; Holota, S.; Zaprutko, L.; Gazell, A. Eur. J. Med. Chem. 2007,42, 641 . http://dx.doi.org/10.1016/j.ejmech.2006.12.006

2. Raj, T.; Bhatia, R. K.; Kapur, A.; Sharma, M.; Saxena, A. K.; Ishar, M. P. S. Eur. J. Med. Chem. 2010, 45,790. http://dx.doi.org/10.1016/j.ejmech.2009.11.001

3. Drews, J. Drug discovery 2000, 287, 1960.

4. Staben, S.T.; Heffron, T. P.; Sutherlin, D. P.; Bhat, S. R.; Castanedo, G. M.; Chuckowree, I. S.; Dotson, J.; Folkes, A. J.; Friedman, L. S.; Lee, L.; Lesnick, J.Bioorg.Med.Chem. Lett. 2010, 20, 6048. http://dx.doi.org/10.1016/j.bmcl.2010.08.067

5. Manetti, D.; Brullo, C.; Magnani, M.; Mosci, F.; Chelli, B.; Crespan, E.; Schenone, S.; Naldini, A.; Bruno, O.; Trincavelli, M. L. J. Med. Chem. 2008, 51, 1252. http://dx.doi.org/10.1021/jm701240c

6. Radi, M.; Dreassi, E.; Brullo, C.; Crespan, E.; Tintori, C.; Brenardo,V.; Valoti, M.; Zamperini,C.; Daigi, H.; Musumeci, F.; Carraro, F. J. Med. Chem.2011, 54, 2610. http://dx.doi.org/10.1021/jm1012819

7. Elgemie, G. H.; Elghandour, A. H.; Elzanti, S. M.; Ahmed, S. A. J. Chem. Res. S,1998, 163165.

8. Catalano, J. G.; Gaitonde, V.; Beesu, M.; Leivers, A. L.; Shotwell, J. B. Tetrahedron Lett.2015,56, 6077. http://dx.doi.org/10.1016/j.tetlet.2015.09.068

9. Zhao, Y. H.; Li, Y.; Guo, T.; Tang, Z.; Xie, W.; Zhao, G. Tetrahedron Lett. 2016, 57, 2257. http://dx.doi.org/10.1016/j.tetlet.2016.04.037

10. Shekarrao, K.; Kaishap, P. P.; Gogoi, S.; Gogoi, S.; Boruah, R. C. Tetrahedron Lett.2014, 55,5251 . http://dx.doi.org/10.1016/j.tetlet.2014.07.119

11. Saikia, P.; Kaishap, P. P.; Prakash, R.; Shekarrao, K.; Gogoi, S.; Boruah, R.C. Tetrahedron Lett.2014,55, 3896.

http://dx.doi.org/10.1016/j.tetlet.2014.05.021

12. Kandeel, M. M.; Abdelall, E. K. A.; Abdelhamid, M. K.; Abdelgwad, M. A.; Philoppes, J. N. Journal of chemical and pharmaceutical research 2013, 5,16. 
13. Hutchinson, I.; Chua, M.-S.; Browne, H. L.; Trapani, V.; Bradshaw, T. D.; Westwell, A. D.; Stevens, M.F.G. J. Med. Chem. 2001,44, 1446. http://dx.doi.org/10.1021/jm001104n

14. Shi, D.-F.; Bradsaw, T. D.; Wrigley, S.; McCall, C.J.; Lelieveld, P.; Fichtner, I.; Stevens, M.F.G. J. Med. Chem. 1996, 39,3375. http://dx.doi.org/10.1021/jm9600959

15. Bradsaw, T. D.; Wrigley, S.; Shi, S. D. -F, Schuitz, R. J.; Paull, K. D.; Stevens, M. F. G. Br. J. Cancer 1998, 77, 745. http://dx.doi.org/10.1038/bjc.1998.122

16. Abdelall E. K. A.; Kamel G. M. Eur. J. Med. Chem. 2016, 118, 250. http://dx.doi.org/10.1016/j.ejmech.2016.04.049

17. Abdelhamid, A. O.; Abdelall, E. K. A.; Abdel-Riheem N. A.; Ahmed, S. A. Phosphorus, Sulfur, Silicon and Relat. Elements2010, 185, 709. http://dx.doi.org/10.1080/10426500902922933

18. Elgemeie, G. H.; El-Enany, M. M.; Ismail, M. M.; Abelall E. K. A. Nucleosides, Nucleotides and Nucleic Acids 2002, 21, 477. http://dx.doi.org/10.1081/NCN-120014820

19. Kandeel, M. M.; Kamal, A. M.; Abdelall, E. K. A.; Elshemy, H. A. H. Eur. J. Med. Chem. 2013, 59, 183 . http://dx.doi.org/10.1016/j.ejmech.2012.11.011

20. Kandeel, M. M; Abdelhameid, M. K.; Abdelall, E. K. A.; Labib, M. B. Chem. Pharm. Bull. 2013, 61, 637. http://dx.doi.org/10.1248/cpb.c13-00089

21. Abdelall, E. K. A.; Mahmoud, A. M.; Abdelhamid, A. O. Phosphorus, Sulfur and Silicon and Related Elements 2010, 185,1862. http://dx.doi.org/10.1080/10426500903348013 